\title{
Dipole Moment of Poly(methylphenylsiloxane) and Copolymers
}

\author{
C. Salom, J. J. Freire, and I. Hernández-Fuentes* \\ Departamento de Quimica Fisica I, Facultad de Ciencias Quimicas, \\ Universidad Complutense, 28040 Madrid, Spain
}

(Received March 14, 1988)

\begin{abstract}
The dielectric constant and the refractive index of several poly(methylphenylsiloxane) fractions $\left(1.17 \times 10^{5} \leqslant \bar{M}_{w} \leqslant 4.56 \times 10^{5}\right)$ with well known tacticity and two copolymers of (dimethylsiloxane-methylphenylsiloxane) $\left(4,200 \leqslant \bar{M}_{n} \leqslant 6,400\right)$ were measured in cyclohexane solution, and in the case of the copolymers also in the bulk state. The copolymer compositions calculated by V-UV spectrometry were $54.3 \%$ and $9.5 \%$, in (methylphenyl)siloxane content. A rotational isomeric state model was used to calculate the dipole moment ratio of the homopolymer. The theoretical value is in good agreement with the experimental one. A comparison of our results with those corresponding to other substituted polysiloxanes shows a very small variation of the dipole moment ratio with the nature of the substituent. The smaller values obtained for the dipole moment ratio of the copolymers in comparison with that of the homopolymer, could be attributed to the short chain length of the copolymers.
\end{abstract}

KEY WORDS Poly(methylphenylsiloxane) / Conformation / Dielectric Constant / Dipole Moment / Dipolar Ratio /

Solution properties of cyclic and linear poly(dimethylsiloxane)s have been widely studied. ${ }^{1-14}$ Theoretical and experimental works about other polysiloxanes (represented at $\left.\left.+\mathrm{RR}^{\prime} \mathrm{SiO}\right\}_{x}\right)$ with substituent groups $\mathrm{R}$ and $R^{\prime}$ aliphatic ${ }^{15-17}$ or aromatic ${ }^{18-21}$ have been also reported. Bibliografic results show that in polysiloxane chains, the nature of the side groups, $R$ and $R^{\prime}$ (at least in symmetrically substituted chains) generally has not a very pronounced effect on the statistical properties of the chain such as dipole moment, as indicated by the values of the dipole moment ratio, $\left\langle\mu^{2}\right\rangle / \mathrm{nm}^{2}$, of poly(dimethylsiloxane) (PDMS) and poly(diethylsiloxane) (PDES) in cyclohexane at $25^{\circ} \mathrm{C}^{13,17}$

In the case of chains with asymmetric centers, however, experimental studies have been so far restricted to samples with unknown tacticity $^{18}$ that introduces a certain degree of ambiguity in the conclusions.

In this work the experimental and theoretical dipole moment of poly(methylphenylsiloxane) (PMPS) samples of well defined tacticity has been determined in order to study the influence the change of a methyl substituent by a phenyl substituent has on the dipole moment of polysiloxane chains.

Moreover the influence of the composition on the dipole moment ratio of copolymers has been also studied using samples of (dimethylsiloxane-methylphenylsiloxane) copolymers in cyclohexane solution. The properties of these copolymers of fluid nature in their bulk state have been also investigated.

\section{EXPERIMENTAL}

\section{Polymers}

The polymers studied in this work have been

\footnotetext{
* To whom all correspondence should be addressed.
} 
poly(methylphenylsiloxane), PMPS, and two copolymers of (dimethylsiloxane-methylphenylsiloxane), that are called cop. 8241 and cop. 8240 .

The initial sample of PMPS was generously provided by Dr. M. A. Llorente from UNED (Spain) and was fractionated before using at $25^{\circ} \mathrm{C}$, in the solvent-non solvent system toluene-methanol. The fractions selected for the dielectric measurements (PMPS-2, PMPS3 and PMPS-5) were characterized by GPC and light scattering, LS. In GPC, silica gel columns and THF as eluent were used. The universal calibration concept with PS standards was used to determine averages of molecular weight. Light scattering was performed on a Fica 42000 instrument at $546 \mathrm{~nm}$. Good Zimm diagrams were obtained in cyclohexane, from which $\bar{M}_{w}$ and the second virial coefficient, $A_{2}$, were calculated. The results are given in Table I.

The tacticity of one of the fractions (PMPS5) and also of the initial sample was determined by ${ }^{1} \mathrm{H}$ NMR. ${ }^{22}$ The following triad percentages were found: 27 isotactic, 49 heterotactic and 24 syndiotactic.

The copolymers, cop. 8240 and cop. 8241 were provided by Polysciences Ltd. Both samples are fluid and their molecular weights are below the limit of LS sensitivity. Their number-average molecular weight was determined with a Knauer vapor pressure osmometer. The copolymer compositions were calculated by two methods: a) by ${ }^{1} \mathrm{H}$ NMR b) by V-UV spectrometry, assuming that the molar absorbance is the same in dilute cyclohexane solution for the homopolymer and the two copolymers. The latter method gives values of the content in methylphenyl siloxane expressed by the molar fraction in the copolymer, $X_{\mathrm{MP}}$, slightly different from the former one and was considered the most accurate since the relaxation rate of the different polymer protons may yield no quantitative ${ }^{1} \mathrm{H}$ NMR spectra. The results are given in Table II.
Table I. Molecular weights, polydispersities and second virial coefficients PMPS fractions

\begin{tabular}{cccc}
\hline Fraction & PMPS-2 & PMPS-3 & PMPS-5 \\
\hline $\bar{M}_{w} \times 10^{-5}(\mathrm{LS})$ & 4.56 & 4.31 & 1.17 \\
$\bar{M}_{w} \times 10^{-5}(\mathrm{GPC})$ & 4.47 & 4.04 & 1.06 \\
$r=\bar{M}_{w} / \bar{M}_{n}(\mathrm{GPC})$ & 2.53 & 2.04 & 1.76 \\
$A_{2} \times 10^{4}$ & 1.64 & 1.59 & 1.74 \\
$\left(\mathrm{~cm}^{3} \mathrm{~g}^{-2} \mathrm{~mol}\right)$ & & & \\
\hline
\end{tabular}

Table II. Copolymer compositions (expressed as molar fraction of methylphenyl siloxane in the copolymer, $X_{\mathrm{MP}}$ ) and number average molecular weights

\begin{tabular}{cccc}
\hline Sample & $X_{\mathrm{MP}}^{\mathrm{UV}}$ & $X_{\mathrm{MP}}^{(\mathrm{NMR})}$ & $\bar{M}_{n}$ \\
\hline Cop. 8241 & 0.543 & 0.431 & 4,200 \\
Cop. 8240 & 0.095 & 0.026 & 6,400 \\
\hline
\end{tabular}

\section{Solvents}

Cyclohexane $(\mathrm{Ch})$, benzene $(\mathrm{Bz})$, and toluene (T1), all of them Carlo Erba RPE, were dried over $4 \AA$ molecular sieves before use.

\section{Dielectric Measurements}

The dielectric constant measurements of cyclohexane polymer solutions were performed on a Multidekameter DK 06 operating at a frequency of $2.0 \mathrm{MHz}$ with a two-terminal cylindrical, glass-silvered cell. The accuracy in the dielectric constant increments was higher than $2 \%$. The apparatus was calibrated using substances with well known dielectric constant (benzene, toluene, and cyclohexane ${ }^{23,24}$ ). Temperature was maintained at $25 \pm 0.05^{\circ} \mathrm{C}$ in all the cases.

In order to verify that at a frequency of $2.0 \mathrm{MHz}$ the polymer molecules are far from the region where the dipole orientation relaxation phenomena start, dielectric constant measurements of several cyclohexane PMPS-3 solutions were carried out in all the range of the apparatus frequencies from 200 to $2500 \mathrm{kHz}$. The same measurements were also performed on a capacitance bridge (General Radio 1620A) and a three terminal platinum cell in the range $0.5-100 \mathrm{kHz}$. The results 
showed that the dependence of dielectric constant, $\varepsilon$, on the polymer weight fraction, $w_{2}$, is insensitive to the measurement frequency in the wide range studied (differences between the values of $\left(\mathrm{d} \varepsilon / \mathrm{d} w_{2}\right)$ in the range $0.5 \mathrm{kHz}-2.5$ $\mathrm{MHz}$ are less than $1 \%$ ). Therefore, it seems apparent that no relaxation phenomena are present at $2.0 \mathrm{MHz}$.

Some preliminary measurements were carried out also with the PMPS-5 fraction in order to determine the best range of concentrations. The dependence of the dielectric constant increment of the solution with respect to that of the pure cyclohexane, $\Delta \varepsilon$, on the polymer weight fraction, $w_{2}$, is shown in Figure 1. It can be observed that this dependence is linear in all the range of the experimental data. Given these results, several cyclohexane solutions of each fraction of PMPS and both copolymers with concentration ranging from $w_{2}=0.003$ to $w_{2}=0.04$ were prepared by dilution of an initial concentrated solution.

\section{Refractive indices}

The increment of the refractive index of the solution with respect to that of the pure solvent, $\Delta n$, was measured on a Brice Phoenix Model $2000 \mathrm{~V}$ differential refractometer at wavelenght of $\lambda=546 \mathrm{~nm}$. The refractive index of pure solvent was taken from the literature. ${ }^{25}$

\section{EXPERIMENTAL RESULTS}

The experimental dipole moments of PMPS and copolymers chains in cyclohexane were obtained by the Guggenheim-Smith equation in its most general form. ${ }^{26,27}$

$$
\begin{aligned}
\frac{4}{3} \pi N_{\mathrm{A}}\left(\alpha_{a_{2}}-\alpha_{a_{2}}^{\prime}+\frac{\left\langle\mu^{2}\right\rangle}{3 k T}\right) & \\
= & \frac{3 M_{2}}{\rho_{1}\left(\varepsilon_{1}+2\right)^{2}}\left[\left(\frac{\mathrm{d} \varepsilon}{\mathrm{d} w_{2}}\right)^{\circ}-\left(\frac{\mathrm{d} n^{2}}{\mathrm{~d} w_{2}}\right)^{\circ}\right]
\end{aligned}
$$

where $\alpha_{a_{2}}$ is the atomic polarizability of the solute obtained in this work according to the equation given by Dasgupta and Smith for poly(dimethylsiloxane), ${ }^{10} \alpha_{a_{2}}^{\prime}$ is the apparent atomic polarizability of the solute, calculated from the apparent molar atomic polarization, $P_{a_{2}}^{\prime}$, through the equation:

$$
P_{a_{2}}^{\prime}=\frac{4}{3} \pi N_{\mathrm{A}} \alpha_{a_{2}}^{\prime}=\left\{\frac{\varepsilon_{1}-1}{\varepsilon_{1}+2}-\frac{n_{1}^{2}-1}{n_{1}+2}\right\} \bar{V}_{2},
$$

$\left\langle\mu^{2}\right\rangle$ is the mean-square dipole moment, $N_{\mathrm{A}}$ is the Avogadro number, $k$ is the Boltzmann constant, $T$ is the absolute temperature, $\rho_{1}$ is the solvent density, $\varepsilon$ and $\varepsilon_{1}$ are the dielectric constants of solution and pure solvent, respectively, $w_{2}$ is the solute weight fraction, $n$ is the solution refractive index, $M_{2}$ is the polymer molecular weight and $\bar{V}_{2}$ is the polymer molar volume. Superscript o means magnitude extrapolated to infinite dilution. The results were expressed as the dipole moment ratio, $\left\langle\mu^{2}\right\rangle / \mathrm{nm}^{2}$, where $n$ is the number of skeletal bonds and $m$ is the dipole moment of the skeletal bonds. In our case we approximated the values of $m$ for PMPS and the copolymers by that of PDMS $(m= \pm 0.60 \mathrm{D})^{11,12}$ neglecting the contribution of phenyl groups; this approximation was used previously for other siloxanes of different substituents. ${ }^{17}$

In Figures 1 and 2, the measured dielectric constants are plotted against the weight fraction, $w_{2}$, for different fractions of the homopolymer and for the copolymers in solution, respectively.

Table III shows the values of $\left(\mathrm{d} n / \mathrm{d} w_{2}\right)^{\circ}$ $\left(\mathrm{d} \varepsilon / \mathrm{d} w_{2}\right)^{\circ}$ and the real, $P_{a_{2}}$, and the apparent, $P_{a_{2}}^{\prime}$, atomic polarization of the solute for the homopolymer and copolymers in cyclohexane at $25^{\circ} \mathrm{C}$ as well as the values of dipole moment ratio, calculated according to the Guggenheim-Smith method. The dipole moment ratio for PMPS-5 was also determined by the Halverstadt-Kumler equation, ${ }^{28}$ which includes the influence of concentration dependence on solution density. The value of the dipole moment ratio obtained was 0.31 , using the measured value of $\left(\mathrm{d} v / \mathrm{d} w_{2}\right)^{\circ}=-0.409$ $\mathrm{cm}^{3} \mathrm{~g}^{-1}$, where $v$ is the specific volume of the 


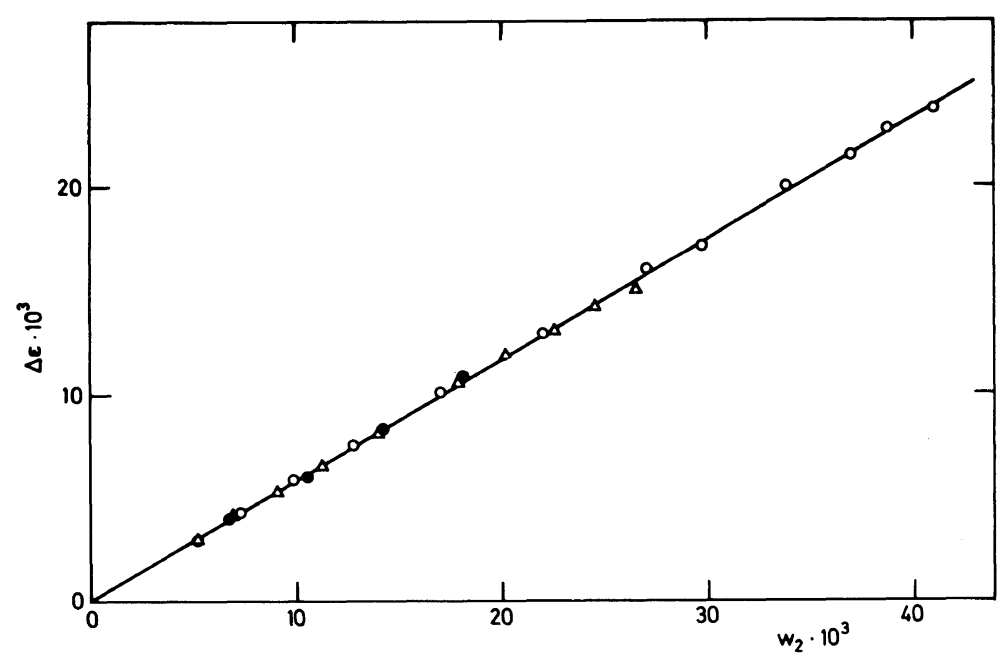

Figure 1. Dielectric constant increment against $w_{2}$ (weight fraction of polymer) for $(\bigcirc)$ PMPS-5, $(\triangle)$ PMPS-2, and (O) PMPS-3, at $25^{\circ} \mathrm{C}$.

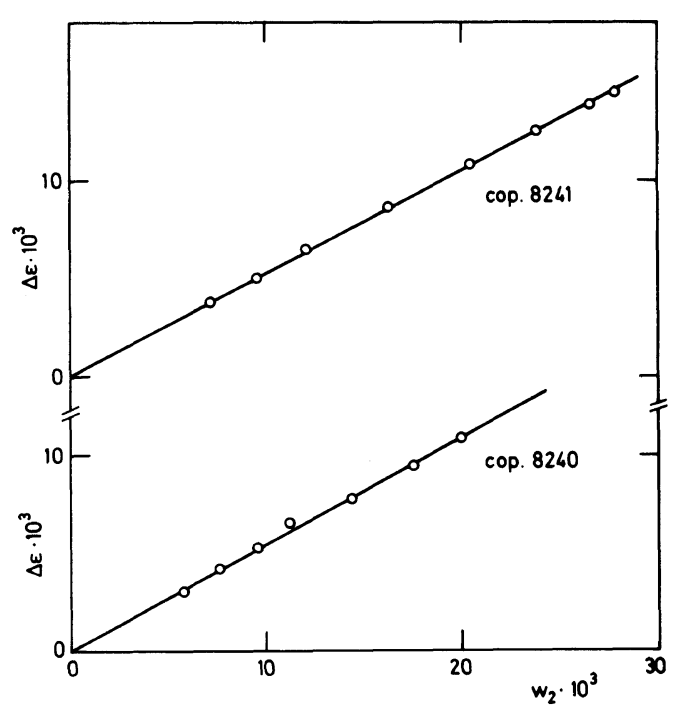

Figure 2. Dielectric constant increment against $w_{2}$ (weight fraction of polymer) for cop. 8240 and cop. 8241 , at $25^{\circ} \mathrm{C}$.

polymer solution. Good agreement between results from the Guggenheim-Smith and Halverstadt-Kumler equations legitimates the elimination of density measurements.

The dipole moments of both copolymers in the bulk state were calculated from the Onsager equation ${ }^{29}$

$$
\begin{aligned}
\left\langle\mu^{2}\right\rangle= & \left(9 k T M v / 4 \pi N_{\mathrm{A}}\right) \\
& \times\left[\left(\varepsilon-n^{2}\right)\left(2 \varepsilon+n^{2}\right) / \varepsilon\left(n^{2}+2\right)^{2}\right]
\end{aligned}
$$

where $\varepsilon, n$, and $v$ are the dielectric constant, the refractive index and the specific volume of the pure copolymer, and $M$ is the copolymer molecular weight (see Table IV). The atomic polarization was also obtained by the DasguptaSmyth equation. The results for the copolymers dipole moment ratio are also included in Table IV.

\section{THEORETICAL RESULTS}

The dipole moment ratios of PMPS of different lengths chains were theoretically calculated by the usual scheme for the evaluation of equilibrium conformational averages. ${ }^{30}$ In these calculations, we employed the rotational isomeric state model with the molecular parameters chosen by Mark and $\mathrm{Ko}^{31}: \theta_{\mathrm{Si}-\mathrm{O}-\mathrm{Si}}=$ $37^{\circ} ; \theta_{\mathrm{O}-\mathrm{Si}-\mathrm{O}}=70.5^{\circ}$. The dipole moment of each repeating unit is on the $\mathrm{Si}-\mathrm{O}$ bond. This is an approximation consistent with our assumption of the dipole moment for skeletal bonds similar to those of PDMS. A single set of three rotational isomeric angles was considered for 
Table III. Values of $\left(\mathrm{d} n / \mathrm{d} w_{2}\right)^{\circ},\left(\mathrm{d} \varepsilon / \mathrm{d} w_{2}\right)^{\circ},\left\langle\mu^{2}\right\rangle / \mathrm{nm}^{2}, P_{a_{2}}$, and $P_{a_{2}}^{\prime}$ for poly(methylphenylsiloxane) and copolymers in cyclohexane at $25^{\circ} \mathrm{C}$

\begin{tabular}{lccrrc}
\hline \multicolumn{1}{c}{ Sample } & $\left(\mathrm{d} n / \mathrm{d} w_{2}\right)^{\mathrm{o}}$ & $\left(\mathrm{d} \varepsilon / \mathrm{d} w_{2}\right)^{\mathrm{o}}$ & \multicolumn{1}{c}{$P_{a_{2}{ }^{\mathrm{a}}}$} & $-P_{a_{2}}^{\prime \mathrm{a}}$ & $\left\langle\mu^{2}\right\rangle / \mathrm{nm}^{2}$ \\
\hline PMPS-2 & 0.0889 & 0.576 & 18,954 & 1,632 & 0.30 \\
PMPS-3 & 0.0889 & 0.580 & 17,915 & 1,458 & 0.31 \\
PMPS-5 & 0.0894 & 0.578 & 4,869 & 299 & 0.31 \\
Cop. 8241 & 0.0519 & 0.517 & 234 & 11 & 0.22 \\
Cop. 8240 & 0.0049 & 0.537 & 461 & 19 & 0.27 \\
\hline
\end{tabular}

a $P_{a_{2}}$ and $P_{a_{2}}^{\prime}$ expressed in $\mathrm{cm}^{3} \mathrm{~mol}^{-1}$.

Table IV. Dielectric constants, refractive indices, specific volumes and dipole moment ratios for cop. 8241 and cop. 8240 at $25^{\circ} \mathrm{C}$

\begin{tabular}{ccccc}
\hline Sample & $\varepsilon$ & $n$ & $v$ & $\left\langle\mu^{2}\right\rangle / \mathrm{nm}^{2}$ \\
\hline Cop. 8241 & 2.896 & 1.4967 & 0.94267 & 0.28 \\
Cop. 8240 & 2.767 & 1.4233 & 1.01650 & 0.30 \\
\hline
\end{tabular}

all units: $\psi_{t}=0^{\circ}, \psi_{g^{+}}=120^{\circ}, \psi_{g^{-}}=-120^{\circ}$. The statistical weights are given by

$$
\begin{gathered}
\boldsymbol{U}_{2}=(1, \sigma, \sigma) \\
\boldsymbol{U}_{\mathrm{d}}=\boldsymbol{U}_{1}=\left(\begin{array}{ccc}
1 & \sigma & \sigma \\
1 & \sigma & 0 \\
1 & 0 & \sigma
\end{array}\right) \\
\boldsymbol{U}_{11}=\left(\begin{array}{ccc}
\omega^{\prime} \omega^{\prime \prime} & \sigma \omega^{\prime \prime} & \sigma \omega^{\prime \prime} \delta \\
\omega^{\prime \prime} \delta & \sigma \delta & \sigma \omega \omega^{\prime} \\
\omega^{\prime \prime} & \sigma \omega \omega^{\prime \prime \prime} & \sigma \delta
\end{array}\right) \\
\boldsymbol{U}_{\mathrm{d} 1}=\left(\begin{array}{ccc}
\left(\omega^{\prime \prime}\right)^{2} & \sigma \omega^{\prime \prime \prime} \delta & \sigma \omega^{\prime} \\
\omega^{\prime \prime \prime} \delta & \sigma & \sigma \omega \omega^{\prime \prime} \\
\omega^{\prime} & \sigma \omega \omega^{\prime \prime} & \sigma \delta^{2}
\end{array}\right)
\end{gathered}
$$

where the rows and columns contain the statistical weights indexed in the order $t, g^{+}, g^{-}$ corresponding to the rotational isomers associated with different bond pairs. The conformational energies associated with the statistical weights are: $E_{\sigma}=0.35 ; E_{\omega}=1.60 ; \quad E_{\omega^{\prime}}=$ $-2.50 ; E_{\omega^{\prime \prime}}=-0.90 ; \quad E_{\omega^{\prime \prime \prime}}=-0.50$, and $E_{\delta}=$ -1.00 in $\mathrm{kcal} \mathrm{mol}^{-1}$. We incorporated fluctuating rotational angles. ${ }^{32,33}$ This modification to the model seems necessary since PMPS exhibits a closed "all trans" confor-
Table V. Theoretical values of the $\left\langle\mu^{2}\right\rangle / \mathrm{nm}^{2}$ of PMPS for different tacticities at $30^{\circ} \mathrm{C}$

\begin{tabular}{cl}
\hline$\%$ meso diads & \multicolumn{1}{c}{$\left\langle\mu^{2}\right\rangle / \mathrm{nm}^{2}$} \\
\hline 0 & 0.2378 \\
0.5 & $0.2679 \pm 0.0007^{\mathrm{a}}$ \\
1.0 & 0.1924 \\
\hline
\end{tabular}

a Statistical average error over different stereosequences.

mation clearly predominant in the particular case of the isotactic chains so that small oscillations in the rotational angles may have important influence on macroscopic properties. ${ }^{19}$ Table $\mathrm{V}$ shows the estimated long chain limit values of these ratios for chains with different tacticity characteristics.

\section{DISCUSSION}

The values of $\left\langle\mu^{2}\right\rangle / \mathrm{nm}^{2}$ are insensitive to the chain length within the range of molecular weights studied in the present work. This can be explained since the dependence of dielectric constant and refractive index increment on the weight fraction of homopolymer are insensitive to molecular weight (see Figure 1 and Table III). These results indicate the absence of excluded volume effects on the dipole moment of the polymer. ${ }^{34-39}$

The dipole moment ratio of PMPS is relatively small. Trans sequences are preferred in the siloxane chains (as it is clearly inferred from theoretical results of distribution functions of the end to end distances ${ }^{32}$ ), and the fact that skeletal bond angles alternate in value 
between 110 and $143^{\circ}$ in these molecules indicates that the all-trans conformation forms a closed structure of small dipole moment. ${ }^{30}$ As can readily be seen, there is good agreement between the experimental and theoretical values of the magnitude $\left\langle\mu^{2}\right\rangle / \mathrm{nm}^{2}$ for the PMPS chains. The theoretical results are not very sensitive to small changes in temperature so that the ones presented here are comparable to the experimental values at $25^{\circ} \mathrm{C}$.

It is also of interest to compare values of the dipole moment ratios for different polysiloxanes. The corresponding ones for poly(dimethylsiloxane) and poly(diethylsiloxane) are 0.37 , and 0.34 respectively. It can be observed that PMPS has a dipole moment ratio similar to that of PDMS and PDES. Therefore, the substitution in a siloxane chain of an alkyl side group for a phenyl group yields very similar values of $\left\langle\mu^{2}\right\rangle / \mathrm{nm}^{2}$, though the intramolecular interactions change significantly (as a consequence of a particularly strong energy preference for $\mathrm{C}_{6} \mathrm{H}_{5} \cdots \mathrm{C}_{6} \mathrm{H}_{5}$ interactions ${ }^{31}$ ). In all the cases the difference between them is less than $20 \%$.

The differences between the values obtained for the dipole moment ratio of the copolymers and those of the homopolymers could be attributed to the short chain length in the copolymers, whose end groups can contribute to the properties of the chain molecule.

The similarities between the values of the dipole moment ratio for chains of both copolymers obtained in solution and in the undiluted state suggest the absence of significant specific solvent effects.

Acknowledgements. Thanks are due to Dr. Julio Guzmán Perote (Instituto de Plásticos y Caucho, C.S.I.C.) for the vapor pressure osmometry measurements Financial support from CAYCIT, Spain, under grant number $0933 / 81$ is gratefully acknowledged.

\section{REFERENCES}

1. K. Dodgson and J. A. Semlyen, Polymer, 18, 1265 (1977).

2. K. Dodgson, D. Sympson, and J. A. Semlyen, Polymer, 19, 1285 (1978).

3. J. S. Higgins, K. Dodgson, and J. A. Semlyen, Polymer, 20, 553 (1979).

4. K. Dodgson, D. J. Bannister, and J. A. Semlyen, Polymer, 21, 663 (1980).

5. C. J. C. Edwards, R. F. T. Stepto, and J. A. Semlyen, Polymer, 21, 781 (1980); ibid., 23, 865 (1982); ibid., 23, 869 (1982).

6. C. J. C. Edwards, D. Rigby, R. F. T. Stepto, K. Dodgson, and J. A. Semlyen, Polymer, 24, 391 (1983); ibid., 24, 395 (1983).

7. M. S. Beevers, S. J. Mumby, S. J. Clarson, and J. A. Semlyen, Polymer, 24, 1565 (1983).

8. R. W. Richards, R. F. T. Stepto, K. Dodgson, J. S. Higgins, and J. A. Semlyen, Polymer, 25, 365 (1984).

9. S. J. Clarson, K. Dodgson, and J. A. Semlyen, Polymer, 26, 930 (1985).

10. S. Dasgupta and C: P. Smyth, J. Chem. Phys., 47, 2911 (1967).

11. C. Sutton and J. E. Mark, J. Chem. Phys., 54, 5011 (1971).

12. J. E. Mark, J. Chem. Phys., 49, 1398 (1968).

13. S. C. Liao and J. E. Mark, J. Chem. Phys., 59, 3825 (1973).

14. M. A. Llorente, A. M. Rubio, and J. J. Freire, Macromolecules, 17, 2307 (1984).

15. C. L. Lee and F. A. Emerson, J. Polym. Sci., 5, 829 (1967).

16. R. R. Buch, H. M. Klimisch, and O. K. Johannson, J. Polym. Sci., 7, 563 (1969).

17. R. R. Rahalkar, D. S. Chiu, and J. E. Mark, J. Chem. Phys., 69, 938 (1978).

18. R. R. Buch, H. M. Klimisch, and O. K. Johannson, J. Polym. Sci., 8, 541 (1970).

19. J. J. Freire and A. M. Rubio, J. Chem. Phys., 81, 2212 (1984).

20. M. R. Gómez-Antón, A. Horta, and I. HernándezFuentes, Polym. Commun., 27, 5 (1986).

21. F. Mendicutti, M. P. Tarazona, and E. Saiz, Polym. Bull., 13, 263 (1985).

22. M. A. Llorente, I. F. Piérola, and E. Saiz, Macromolecules, 18, 2663 (1985).

23. A. J. Petro and C. P. Symth, J. Am. Chem. Soc., 79, 6142 (1957).

24. W. M. Heston and C. P. Smyth, J. Am. Chem. Soc., 72, 99 (1950).

25. M. B. Huglin, "Light Scattering from Polymer Solutions," Academic Press, London, 1972.

26. E. A. Guggenheim, Trans. Faraday Soc., 45, 714 (1949); ibid., 47, 573 (1951).

27. J. W. Smith, Trans. Faraday Soc., 46, 394 (1950). 
28. I. F. Halverstadt and W. D. Kumler, J. Am. Chem. Soc., 64, 2988 (1942).

29. L. Onsager, J. Am. Chem. Soc., 58, 1486 (1936).

30. P. J. Flory, "Statistical Mechanics of Chain Molecules," Wiley, New York, N. Y., 1969.

31. J. E. Mark and J. H. Ko, J. Polym. Sci., 13, 2221 (1975).

32. J. J. Freire and M. Fixman, J. Chem. Phys., 69, 634 (1978).

33. R. Cook and M. Moon, Macromolecules, 13, 1537
(1980).

34. J. Marchal and H. Benoit, J. Chim. Phys., 52, 818 (1955).

35. J. Marchal and H. Benoit, J. Polym. Sci., 23, 223 (1957).

36. R. J. W. Le Fèvre and K. M. Sundaran, J. Chem. Soc., 3188 (1963).

37. W. H. Stockmayer, Pure Appl. Chem., 15, 539 (1967).

38. K. Nagai and T. Ishikawa, Polym. J., 2, 416 (1971).

39. M. Doi, Polym. J., 3, 252 (1972). 